\title{
Risk Prediction and Treatment of LE-DVT in Patients with Chronic Radiation Intestinal Injury: A Retrospective Case-Control Study
}

\author{
Xiaoyan Huang, ${ }^{1-3, *}$ Yingyi \\ Kuang, ${ }^{1-3, *}$ Qiyuan Qin, ${ }^{1-3, *}$ \\ Miaomiao Zhu, ${ }^{1-3}$ Yanjiong \\ $\mathrm{He},{ }^{\mathrm{I}-3}$ Zixu Yuan, ${ }^{\mathrm{I}-3}$ Huaiming \\ Wang, (1) ${ }^{1-3}$ Qinghua \\ Zhong, ${ }^{1,2,4}$ Qi Guan, ${ }^{1-3}$ Hui \\ Wang, ${ }^{1-3, *}$ Tenghui Ma, ${ }^{1-3, *}$ \\ Xinjuan Fan $\mathbb{D}^{1,2,5, *}$
}

'Guangdong Institute of Gastroenterology, The Sixth Affiliated Hospital, Sun Yat-sen University, Guangzhou, Guangdong, 510655, People's Republic of China; ${ }^{2}$ Guangdong Provincial Key Laboratory of Colorectal and Pelvic Floor Diseases, The Sixth Affiliated Hospital, Sun Yat-sen University, Guangzhou, Guangdong, 510655, People's Republic of China; ${ }^{3}$ Department of Colorectal Surgery, The Sixth Affiliated Hospital, Sun Yat-sen University, Guangzhou, Guangdong, 510655, People's Republic of China; ${ }^{4}$ Department of Endoscopic Surgery, The Sixth Affiliated

Hospital, Sun Yat-sen University, Guangzhou, Guangdong, 510655, People's Republic of China; ${ }^{5}$ Department of Pathology, The Sixth Affiliated Hospital, Sun Yat-sen University, Guangzhou, Guangdong, 510655, People's Republic of China

*These authors contributed equally to this work

Correspondence: Xinjuan Fan Department of Pathology, Sun Yat-sen University Sixth Affiliated Hospital, No. 26 Yuancun Erheng Road, Guangzhou, 510655, Guangdong, People's Republic of China Fax +86 20-38737621

Email fanxjuan@mail.sysu.edu.cn

Tenghui Ma

Department of Colorectal Surgery, Sun Yat-sen University Sixth Affiliated

Hospital, No. 26 Yuancun Erheng Road, Guangzhou, 5I 0655, Guangdong, People's Republic of China

Fax +86 20-3873762 I

Email matengh@mail.sysu.edu.cn
Background: Chronic radiation intestinal injury (CRII) is the most common complication after pelvic malignancy radiation. Once hemorrhagic CRII patients suffer from lower extremity deep venous thrombosis (LE-DVT), hemostasis and anticoagulation therapy will be adopted simultaneously, but the treatment strategy is a paradox, as the condition is extremely intractable and serious. The aim of this study was to investigate the prevalence of and risk factors for LE-DVT in CRII patients and explore the treatment of hemorrhagic CRII patients with LE-DVT.

Methods: This was a retrospective study, and a total of 608 hospitalized CRII patients after pelvic radiotherapy were included from November 2011 to October 2018. Univariate and multivariate analyses were conducted to investigate the potential risk factors for LE-DVT in CRII patients. Furthermore, the treatment of hemorrhagic CRII patients with LE-DVT was explored.

Results: Among the CRII patients, 94 (15.5\%) were with suspicious symptoms of LE-DVT in the lower limbs, and 32 (5.3\%) were diagnosed with LE-DVT. Among the patients with LE-DVT, 65.6\% (21/32) had bleeding simultaneously, and 29 (90.6\%) had anemia with 24 $(75.0 \%)$ having moderate to severe anemia. Multivariate analysis showed that a recent surgical history ( $\leq 6$ months) $(\mathrm{OR}=5.761,95 \% \mathrm{CI}: 2.506 \sim 13.246, p<0.001)$, tumor recurrence or metastasis $(\mathrm{OR}=3.049,95 \% \mathrm{CI}: 1.398 \sim 6.648, p=0.005)$ and the hemoglobin (Hb) level ( $\mathrm{OR}=0.960,95 \% \mathrm{CI}: 0.942 \sim 0.979, p<0.001)$ were significantly associated with the development of LE-DVT. ROC curve analysis showed that the AUC of the merged risk score of the independent risk factors was 0.822 (95\% CI: 0.789 0.852), and the optimal $\mathrm{Hb}$ cutoff was $82.5 \mathrm{~g} / \mathrm{L}$. After colostomy, obvious bleeding remission was rapidly found in $84.6 \%$ of hemorrhagic CRII patients with LE-DVT.

Conclusion: The prevalence of LE-DVT in hospitalized CRII patients was 5.3\%. A recent surgical history, tumor recurrence or metastasis and a lower $\mathrm{Hb}$ level were independently associated with LE-DVT development in CRII patients. Colostomy could be a good choice for intractable hemorrhagic CRII patients with LE-DVT.

Keywords: chronic radiation intestinal injury, lower extremity deep venous thrombosis, pelvic malignancy radiation, risk factor, colostomy, hemorrhagic

\section{Introduction}

Radiotherapy is among the most effective treatments for pelvic malignancy, with 0.8 2.4 million patients worldwide estimated to require radiotherapy for pelvic cancer in 2020 alone. $^{1-4}$ However, radiation-induced intestinal injury (RII) is an unavoidable side effect and the most common complication of pelvic malignancy 
radiation, damaging the small and/or large intestine. ${ }^{5}$ According to the end time of radiotherapy, RII is divided into acute (less than 3 months after radiotherapy, ARII) and chronic (more than 3 months after radiotherapy, CRII) types. Ninety percent of patients present with ARII, and subsequently, $5 \%$ to $55 \%$ develop CRII post-pelvic radiotherapy. ${ }^{6-8}$ Most of the symptoms of ARII are transient, self-healing, and relieved within 3 months. While CRII often emerges 12 24 months after the end of radiotherapy, it is progressive and difficult to reverse. ${ }^{9,10}$ The main clinical presentations of CRII are intestinal obstruction, diarrhea, bleeding, abdominal cramping, anal pain, perforation, necrosis, abscess, fistulas, strictures, severe anemia, chronic malabsorption, and even death, seriously impairing quality of life. ${ }^{11,12}$ Chronic radiation proctitis (CRP) is another term used to describe the involvement of the rectum and sigmoid colon, with the most prominent characteristic being bleeding, accounting for $>80 \%$ of CRP patients. ${ }^{13,14}$

Lower extremity deep venous thrombosis (LE-DVT) is a common condition and major cause of morbidity and mortality worldwide. LE-DVT lesions can detach and embolize into the heart or lung, causing cardiopulmonary embolism or even death. Numerous studies have demonstrated that DVT is a complex multifactorial disease influenced by several risk factors, including surgery and trauma, prolonged immobilization, myeloproliferative disorders, pregnancy and postpartum status, weight, age, smoking status and corticosteroid treatment. ${ }^{15-17}$ In addition, radiotherapy and cancer increase the risk of developing DVT, ${ }^{18-20}$ and the reported cumulative incidence of venous thrombosis in cancer patients is $1-8 \%{ }^{21}$ Moreover, after radiotherapy, changes in the structure of the pelvic tissue, the blood composition and the vessel wall are more rapid than those of fibrosis, ${ }^{22,23}$ and radionecrosis and granulation of the small lymphatic vessels, lymph nodes, and soft tissue around vessels following radiotherapy can lead to the accumulation of protein-rich fluid in soft tissues secondary to inadequate lymphatic drainage. $^{23-25}$ Given the serious potential consequences of LE-DVT, knowing more about its prevalence in CRII patients is extraordinarily important. In addition, bleeding is a common symptom of CRII. If LE-DVT and bleeding simultaneously occur in one CRII patient, hemostasis and anticoagulation therapy will be conducted, ${ }^{26}$ which is a therapeutic contradiction and will greatly increase the difficulty of treatment.
To the best of our knowledge, studies regarding the occurrence of LE-DVT and risk factors for developing LEDVT in CRII patients are still scarce. Thus, to better prevent and treat CRII complicated with LE-DVT, the incidence of and risk factors for LE-DVT in CRII patients were retrospectively analyzed in this study, and an appropriate treatment strategy was explored.

\section{Materials and Methods \\ Patients}

Hospitalized CRII patients in our hospital from November 2011 to October 2018 were retrospectively included. The diagnostic criteria of CRII were as follows: three months after pelvic malignancy radiation; one or multiple symptoms of intestinal obstruction, diarrhea, bleeding, abdominal cramping, anal pain, perforation, necrosis, abscess, fistulas, strictures, severe anemia, etc.; mucosal fragility, pallor, spontaneous bleeding, telangiectasias, mucosal edema, strictures, fistulas, or ulcerations as observed under endoscopy; and diagnosis by examinations such as magnetic resonance imaging (MRI) and computed tomography (CT). Hospitalized CRII patients needed to satisfy at least one of the following conditions: moderate to severe bleeding evaluated according to the modified Subjective Objective Management Analysis (SOMA, Table 1$)^{27,28}$ or severe complications with intestinal obstruction, perforation, necrosis, abscess, fistulas, strictures, severe anemia (hemoglobin $(\mathrm{Hb})<70 \mathrm{~g} / \mathrm{L}$ ), and chronic malabsorption, etc. Anemia was defined as a $\mathrm{Hb}$ level $<120$ $\mathrm{g} / \mathrm{L}$ for males and $<110 \mathrm{~g} / \mathrm{L}$ for females and categorized into mild anemia $(\mathrm{Hb} \geq 90 \mathrm{~g} / \mathrm{L})$, moderate anemia $(\mathrm{Hb} \geq 70 \sim<0$ $\mathrm{g} / \mathrm{L})$, and severe anemia $(\mathrm{Hb}<70 \mathrm{~g} / \mathrm{L})$, as shown in Table 1. In this study, the inclusion criteria were as follows: diagnosis of CRII; hospitalization mainly for the treatment of CRII; and symptoms of DVT in the lower limbs. The

Table I Modified Subjective/Objective Management Analysis System to Assess the Severity of Bleeding

\begin{tabular}{|l|l|l|l|}
\hline Grade & Bleeding & Severity & Anemia $(\mathbf{H b}, \mathbf{g} / \mathbf{L})$ \\
\hline $\mathrm{I}$ & $\begin{array}{l}\text { Mild } \\
\text { bleeding }\end{array}$ & $\begin{array}{l}\text { Occasional } \\
\text { or occult }\end{array}$ & $\begin{array}{l}\text { Mild anemia }(\mathrm{Hb}: \text { male } \geq \\
90 \sim<120 \mathrm{~g} / \mathrm{L} ; \text { female } \geq \\
90 \sim<110 \mathrm{~g} / \mathrm{L})\end{array}$ \\
\hline 2 & $\begin{array}{l}\text { Moderate } \\
\text { bleeding }\end{array}$ & Persistent & $\begin{array}{l}\text { Moderate anemia }(\mathrm{Hb}: \\
\geq 70 \sim<90 \mathrm{~g} / \mathrm{L})\end{array}$ \\
\hline 3 & $\begin{array}{l}\text { Severe } \\
\text { bleeding }\end{array}$ & Gross & $\begin{array}{l}\text { Severe anemia, }(\mathrm{Hb}:<70 \mathrm{~g} / \mathrm{L}), \\
\text { transfusion needed }\end{array}$ \\
\hline
\end{tabular}




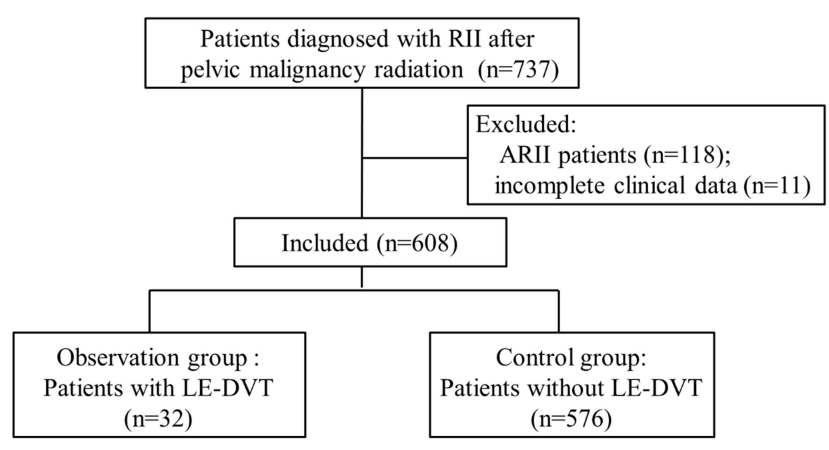

Figure I Flow chart of patient selection.

exclusion criteria were as follows: acute radiation-induced intestinal injury (less than 3 months after radiotherapy, ARII); previous blood-related diseases or severe coagulation disorders; and incomplete clinical data. From November 2011 to October 2018, 737 hospitalized RII patients were screened, and a total of 608 CRII patients were included in this study (Figure 1). The clinical data of each included patient were carefully collected.

\section{Data Collection}

CRII patients with suspicious symptoms of LE-DVT such as edema, and pain in the lower extremity etc. were routinely examined by bilateral lower extremity venous Doppler ultrasonography to identify whether there was LE-DVT or not. Clinical data were compared between patients with LE-DVT (observation group) and patients without LE-DVT (control group). The following data were compared: age, sex, body mass index (BMI), cardiovascular and cerebrovascular diseases, diabetes mellitus, blood transfusion history, smoking status, surgical history, recent surgical history, hemostasis drug use, tumor recurrence or metastasis, time to the end of radiotherapy, corticosteroid treatment, radiotherapy dose, $\mathrm{Hb}$ level, blood platelet count (BPC), mean platelet volume (MPV), white blood cell (WBC) count, and albumin (ALB) level. The clinical data and laboratory values of the patients were collected initially after admission. Then, the risk factors for developing LE-DVT in CRII patients were retrospectively investigated. In addition, CRII patients with bleeding were graded at three levels according to the modified SOMA to assess the severity of bleeding.

\section{Statistical Analysis}

Data analysis in this study was performed using SPSS software, version 19.0 (Chicago, IL, USA). Continuous variables are expressed as the mean $\pm \mathrm{SD}$ or median (interquartile range, IQR) and were compared using the $t$-test or Mann-Whitney $U$-test as appropriate. Categorical variables are expressed as numbers and percentages and were compared using Fisher's test or the $\chi^{2}$ test as appropriate. Variables that were significantly different between groups in the univariate analysis were included in the multivariate logistic regression model in a stepwise manner to assess the associations between potential risk factors and LE-DVT. For the independent risk factors, the best cutoff values for distinguishing between CRII patients with and without LE-DVT were assessed by receiver operating characteristic (ROC) curve analyses. A two-sided $p<0.05$ was considered statistically significant.

This work is reported in line with the STROCSS criteria.

\section{Results}

\section{Demographics and Characteristics}

In this study, the primary cancers of the CRII patients included cervical cancer $(n=460)$, endometrial cancer $(n=29)$, ovarian cancer $(n=7)$, vaginal cancer $(n=4)$, prostate cancer $(n=24)$, testicular cancer $(n=1)$, rectal cancer $(n=77)$, anal cancer $(n=3)$, and colon cancer $(n=1)$. Of the 608 included patients, there were 528 (86.8\%) CRP patients, including 439 (83.1\%) patients with bleeding, and 387 (63.7\%) CRII patients who presented with anemia (shown in Table 2); anemia was significantly associated with thrombocytosis $(p<0.001)$.

Among the 608 patients eligible for this study, 94 (15.5\%) CRII patients had suspicious symptoms of LE-DVT. After confirmation by bilateral lower extremity venous Doppler ultrasonography, 32 patients with LE-DVT were identified. Thus, the prevalence of LE-DVT among all of the CRII

Table 2 Bleeding-Related Data of the Included CRII Patients

\begin{tabular}{|l|c|c|}
\hline n (\%) & $\begin{array}{c}\text { Total in Study } \\
(\mathbf{n}=\mathbf{6 0 8})\end{array}$ & $\begin{array}{c}\text { Total with LE- } \\
\text { DVT (n = 32) }\end{array}$ \\
\hline CRP & $528(86.8 \%)$ & $26(81.3 \%)$ \\
\hline Bleeding & $439(72.2 \%)$ & $21(65.6 \%)$ \\
\hline Severity of bleeding & & \\
Grade I & $272(44.7 \%)$ & $4(12.5 \%)$ \\
Grade 2 & $105(17.3 \%)$ & $15(46.9 \%)$ \\
Grade 3 & $62(10.2 \%)$ & $2(6.3 \%)$ \\
\hline Anemia & $387(63.7 \%)$ & $29(90.6 \%)$ \\
Mild anemia $(\mathrm{Hb} \geq 90 \mathrm{~g} / \mathrm{L})$ & $173(28.5 \%)$ & $5(15.6 \%)$ \\
Moderate anemia $(\mathrm{Hb} 70-$ & $135(22.2 \%)$ & $17(53.1 \%)$ \\
90 g/L) & $79(13.0 \%)$ & $7(21.9 \%)$ \\
Severe anemia $(\mathrm{Hb}<70 \mathrm{~g} / \mathrm{L})$ & &
\end{tabular}

Abbreviation: CRP, chronic radiation proctitis. 
patients hospitalized after pelvic malignancy radiation was $5.3 \%$ (32/608). Among these CRII patients with LE-DVT, 8 cases affected the left lower limb, 13 affected the right lower limb, and 11 affected both lower limbs. The age of these patients was $57 \pm 11$ years old, with 3 males and 29 females; the median time to presentation of LE-DVT was 15 months after radiotherapy. The primary cancers of the CRII patients with LE-DVT included cervical cancer $(n=27)$, endometrial cancer $(n=1)$, testicular cancer $(n=1)$, and rectal cancer $(n=3)$. The incidence of LE-DVT among CRII patients who had ever suffered from cervical cancer was 5.9\% (27/460). In addition, $65.6 \%(21 / 32)$ of patients had rectal bleeding. After grading the hemorrhagic patients with LE-DVT according to SOMA, we found that $12.5 \%(4 / 32)$ of patients had grade 1 bleeding, 46.9\% (15/32) had grade 2 bleeding, and 6.3\% (2/ 32 ) had grade 3 bleeding. Furthermore, the anemic degree of the patients was also evaluated according to the Hb level (see Table 1). There were 29 (90.6\%) anemic patients with LEDVT, with $53.1 \%$ having moderate anemia and $21.9 \%$ having severe anemia (listed in Table 2).

\section{Comparisons of CRII Patients with and without LE-DVT}

The associations of the clinical data of the patients with and without LE-DVT are listed in Table 3 . In the univariate analysis, significantly larger proportions of the patients with a recent surgical history ( $\leq 6$ months) $(31.4 \%$ vs $8.7 \%, p<0.001)$, a history of hemostatic drug use $(53.1 \%$ vs $31.3 \%, p=0.010)$, tumor recurrence or metastasis $(40.6 \%$ vs $17.0 \%, p=0.001)$, corticosteroid treatment (56.3\% vs $26.7 \%, p<0.001)$, an ALB level $\leq 35 \mathrm{~g} / \mathrm{L}(56.3 \%$ vs $24.8 \%, p<0.001)$, an elevated WBC count [(5.8 (4.810.6) vs $5.0(4.0-6.5), p=0.003)]$, and a low Hb level $[(80.2 \pm 16.1$ vs $100.4 \pm 23.2, p<0.001)]$ were found in the observation group than in the control group. In contrast, there was no significant difference in age, BMI, cardiovascular and cerebrovascular disease, diabetes mellitus, surgical history, smoking history, time to the end of radiotherapy, BPC, or MPV between the two groups.

Variables of potential risk factors that were significantly different between the groups were included in the multivariate logistic regression analysis. Table 4 shows that a recent surgical history ( $\leq 6$ months) $(\mathrm{OR}=5.761,95 \% \mathrm{CI}$ : 2.506 13.246, $p<0.001$ ), tumor recurrence or metastasis $(\mathrm{OR}=3.049,95 \% \mathrm{CI}: 1.398 \sim 6.648, p=0.005)$ and the $\mathrm{Hb}$ level (OR=0.960, 95\% CI: 0.942 0.979, $p<0.001$ ) were significantly associated with the occurrence of LE-DVT. The results suggest that a recent surgical history, tumor recurrence or metastasis and a low $\mathrm{Hb}$ level independently increased the risk of LE-DVT in CRII patients.

Table 3 Characteristics of the CRII Patients with or without LE-DVT

\begin{tabular}{|c|c|c|c|}
\hline Variables & Observation Group $(n=32)$ & Control Group $(n=576)$ & $p$ \\
\hline Age $\geq 50$ y, n (\%) & $25(78.1)$ & $438(76.0)$ & 0.788 \\
\hline Male, n (\%) & $3(9.4)$ & $72(12.5)$ & 0.805 \\
\hline $\mathrm{BMI}<\mathrm{I} 8.5 \mathrm{~kg} / \mathrm{m}^{2}, \mathrm{n}(\%)$ & $6(18.8)$ & $146(25.3)$ & 0.382 \\
\hline Cardiovascular and cerebrovascular diseases, $\mathrm{n}$ (\%) & $5(15.6)$ & $95(16.5)$ & 0.897 \\
\hline Diabetes mellitus, n (\%) & $2(6.3)$ & $70(12.2)$ & 0.469 \\
\hline Blood transfusion history, $\mathrm{n}(\%)$ & $7(21.9)$ & $|7|(29.8)$ & 0.344 \\
\hline Smoking status, yes, n (\%) & $0(0)$ & $13(2.3)$ & 0.391 \\
\hline Surgical history, n (\%) & $23(71.9)$ & $345(59.9)$ & 0.177 \\
\hline Recent surgical history ( $\leq 6$ months), $\mathrm{n}(\%)$ & II (34.4) & $50(8.7)$ & 0.000 \\
\hline Hemostatic drug use history, n (\%) & $17(53.1)$ & $180(31.3)$ & 0.010 \\
\hline Tumor recurrence or metastasis, $\mathrm{n}(\%)$ & $13(40.6)$ & $98(17.0)$ & 0.001 \\
\hline Time to the end of radiotherapy $\geq 24$ months, $n$ (\%) & $9(28.1)$ & $190(33.0)$ & 0.568 \\
\hline Corticosteroid treatment, n (\%) & $18(56.3)$ & $152(26.7)$ & 0.000 \\
\hline Radiotherapy dose, Gy, median (IQR) & $79.0(69.5-88.5)$ & $80.0(56.0-86.0)$ & 0.333 \\
\hline $\mathrm{Hb}, \mathrm{g} / \mathrm{L}$ mean $\pm \mathrm{SD}$ & $80.2 \pm 16.1$ & $100.4 \pm 23.2$ & 0.000 \\
\hline $\mathrm{BPC}, \times 10^{9} / \mathrm{L}$, median $(\mathrm{IQR})$ & $241.8(\mid 62.1-387.0)$ & $235.0(\mid 86.0-295.5)$ & 0.668 \\
\hline MPV, fl, mean \pm SD & $8.6 \pm 1.4$ & $9.1 \pm 1.3$ & 0.054 \\
\hline WBC, $\times 10^{9} / \mathrm{L}$, median (IQR) & $5.8(4.8-10.6)$ & $5.0(4.0-6.5)$ & 0.003 \\
\hline ALB $\leq 35 \mathrm{~g} / \mathrm{L}, \mathrm{n}(\%)$ & $18(56.3)$ & $143(24.8)$ & 0.000 \\
\hline
\end{tabular}

Note: Data were expressed as means \pm SDs, medians (IQR) or $n(\%)$.

Abbreviations: BMI, body mass index; IQR, interquartile range; SD, standard deviation; Hb, hemoglobin, BPC, platelet count; MPV, mean platelet volume; WBC, white blood cells; ALB, albumin. 
Table 4 Multivariate Logistic Regression Analysis for Developing LE-DVT in CRII Patients After Pelvic Malignancy Radiation

\begin{tabular}{|l|c|c|c|c|c|}
\hline Variables & Coefficient & Standard Error & OR & 95\% Cl Lower Limit Higher Limit & $\boldsymbol{P}$ \\
\hline Recent surgical history ( $\leq 6$ months) & $1.75 \mathrm{I}$ & 0.425 & 5.761 & $2.506 \sim 13.246$ & 0.000 \\
Tumor recurrence or metastasis & 1.115 & 0.398 & 3.049 & $1.398 \sim 6.648$ & 0.005 \\
$\mathrm{Hb}$ & -0.040 & 0.010 & 0.960 & $0.942 \sim 0.979$ & 0.000 \\
\hline
\end{tabular}

Abbreviations: $\mathrm{OR}$, odds ratio; $\mathrm{Cl}$, confidence interval; $\mathrm{Hb}$, hemoglobin.

\section{ROC Curve of Independent Factors}

ROC curve analysis was used to evaluate the potency of the identified independent factors for predicting the occurrence of LE-DVT in CRII patients and compare the potential prognostic variables (Figure 2). The results showed that the area under the ROC curve (AUC) of the merged risk score for predicting the occurrence of LE-DVT in CRII patients was significantly higher [0.822 (95\% CI: 0.789 0.852)] than that of the other variables, such as a recent surgical history $\leq 6$ months [0.628 (95\% CI: 0.589 0.667), $p<0.0001]$, tumor recurrence or metastasis [0.618 (95\% CI: 0.578 0.657), $p<0.0001]$, and the $\mathrm{Hb}$ level $[0.756 \quad(95 \%$ CI: $0.688 \sim 0.824), p=0.024]$. The cutoff value for the $\mathrm{Hb}$ level was $82.5 \mathrm{~g} / \mathrm{L}$ (sensitivity $=71.9 \%$, specificity $=75.5 \%$ ).

\section{Treatment}

To investigate the treatment strategies adopted for hemorrhagic patients with LE-DVT in this study, hemorrhagic CRII patients with LE-DVT were followed up for at least 1 year. We found that because of uncontrollable bleeding, colostomy was performed in $61.9 \%(13 / 21)$ of hemorrhagic CRII patients with LE-DVT, which was significantly

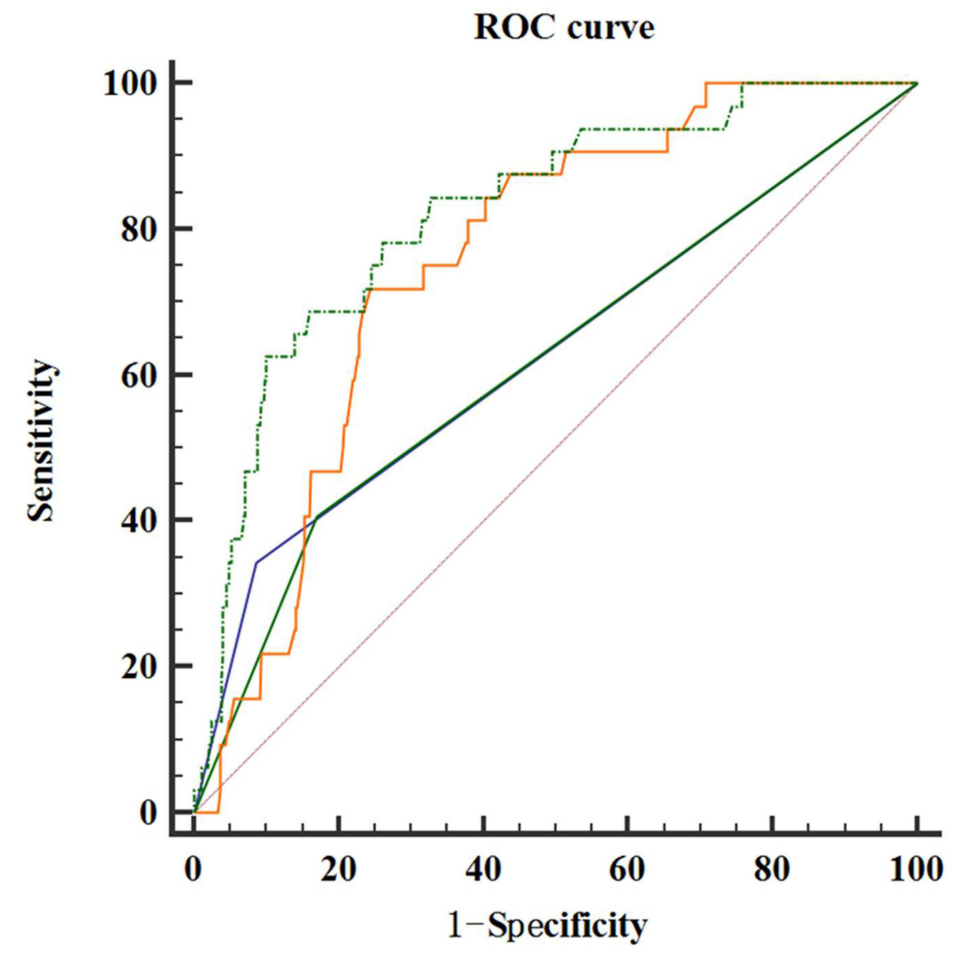

$\begin{array}{lcc} & \text { AUC }(95 \% \mathrm{CI}) & p \\ \text { Recent surgical history } \leq 6 \text { months } & 0.628(0.589 \sim 0.667) & <0.0001 \\ \text { Tumor recurrence or metastasis } & 0.618(0.578 \sim 0.657) & <0.0001 \\ \text { Hb } & 0.756(0.688 \sim 0.824) & 0.024 \\ \text { Merged risk score } & 0.822(0.789 \sim 0.852) & -\end{array}$

Figure 2 ROC curve of independent factors for predicting LE-DVT development in CRII patients after pelvic malignancy radiation and comparison with the potential prognostic variables.

Abbreviations: ROC, receiver operating characteristic; AUC, area under the receiver-operating characteristic curve; Hb, hemoglobin. 
higher than the corresponding proportion of hemorrhagic CRII patients without LE-DVT (17.5\%, 73/418). In addition, among the remaining 8 hemorrhagic CRII patients with LE-DVT, 3 patients underwent bowel resection because of unendurable pain or fistula, and the other 5 received no surgery. Moreover, after colostomy, obvious bleeding remission was rapidly observed in 11 (84.6\%) hemorrhagic CRII patients with LE-DVT, and LE-DVT disappeared or improved in all these patients following anticoagulation therapy or with vena cava filter or stent placement (LE-DVT obviously improved in 2 patients and disappeared in 1 following anticoagulation therapy and was remarkably relieved in 1 patient and cured in 2 patients following vena cava filter or stent placement with anticoagulation therapy). In contrast, in the 5 hemorrhagic CRII patients with LE-DVT treated without colostomy, bleeding existed for at least one year. Unfortunately, the follow-up LE-DVT treatment data from the remaining hemorrhagic CRII patients with LE-DVT were unavailable because there were no ultrasonic examinations after the patients were treated or we could not contact the patient.

\section{Discussion}

In the present study, the prevalence of symptomatic LEDVT after pelvic malignancy radiation in the CRII patients we evaluated was $5.3 \%$, which is much higher than that in the general population because these patients had a history of pelvic radiation therapy and cancer. ${ }^{29}$ In this study, only 94 CRII patients with suspicious symptoms of LE-DVT, such as edema and pain in the lower extremities, were tested to verify the presence of LE-DVT, while other patients may have had LE-DVT but showed no symptoms. It has been reported that occult DVT is also more common in patients with cancer. ${ }^{30}$ Therefore, the occurrence of LEDVT in CRII patients after pelvic malignancy radiation might be underestimated. More attention should be given to identifying and preventing LE-DVT in CRII patients. Therefore, this study investigated the associated risk factors for the development of LE-DVT in CRII patients. A history of hemostatic drug use, an ALB level less than $35 \mathrm{~g} / \mathrm{L}$, an inflammatory cell count (WBC count), and the use of corticosteroids, such as hexadecadrol and budesonide, were obviously associated with the development of LE-DVT in CRII patients. Previously, it has been reported that a history of hemostatic drug use aggravates the hypercoagulable state and hypoalbuminemia and that corticosteroids significantly promote DVT. ${ }^{31}$ Growing evidences have demonstrated that inflammation triggers a variety of responses that lead to increased coagulation and thrombosis. ${ }^{32,33}$ Our study is consistent with the previous studies.

Our results showed that a recent surgical history $(\leq 6$ months) was independently related to the development of LE-DVT in CRII patients, which may be due to prolonged immobilization. It has been reported that surgery and trauma indeed increase the risk of developing DVT, and surgical operations prolong immobilization. ${ }^{16}$ Our results are consistent with those of a previous study. A previous study declared that cancer creates a hypercoagulable state and that the risk of developing DVT is much higher in patients with active cancer. ${ }^{34}$ The incidence of VTE in patients with cancer is estimated to be 4-7 times higher than that in patients without cancer. ${ }^{35,36}$ In this study, we also found that the proportion of tumor recurrence or metastasis in the observation group $(40.6 \%)$ was much higher than that in the control group (17.0\%), and the results showed that tumor recurrence or metastasis was an independent risk factor for LE-DVT in CRII patients.

Moreover, a lower $\mathrm{Hb}$ level was another independent risk factor for LE-DVT in CRII patients. Of the included patients in this study, $86.8 \%$ were CRP patients, and $83.1 \%(439 / 528)$ and $63.6 \%(336 / 528)$ of the CRP patients were hemorrhagic and anemic, respectively. In addition, 90.6\% of the anemic CRP patients had LE-DVT, of whom $75 \%$ had moderate to severe anemia (Table 2). Previously, it has been proven that anemia promotes DVT formation because anemia is considered a hyperkinetic state that disturbs the expression of endothelial adhesion molecule genes and can result in endothelial injuries that subsequently result in thrombus formation. ${ }^{37}$ In this study, the main cause of anemia in patients was hemorrhage, which may give rise to iron deficiency and possibly secondary thrombocytosis and thus lead to an increased thromboembolic risk. ${ }^{38,39}$

In the present study, among the 32 CRII patients with LE-DVT, 21 (65.6\%) presented with bleeding, and 90.6\% presented with anemia. Hemostasis and anticoagulation therapy were needed for the CRII patients suffering from bleeding and LE-DVT simultaneously, which was a paradox and greatly increased the difficulty of treatment. Therefore, detecting LE-DVT in CRII patients earlier and maintaining the treatment balance are extremely important.

It has been reported that if CRII patients suffer from severe intractable bleeding refractory to conservative treatments or require blood transfusions because of bleeding, 
diverting colostomy is a simple, effective and safe procedure that can lead to a higher rate of bleeding remission (94\% vs $12 \%$ ) and obviously increase the $\mathrm{Hb}$ level compared to conservative treatment. ${ }^{28}$ In this study, among the 21 hemorrhagic CRII patients with LE-DVT, 13 patients had recurrent refractory rectal bleeding or even needed blood transfusion; anticoagulation therapies that can aggravate bleeding were cautiously applied and often withdrawn in these patients. Then, these 13 patients underwent colostomy, and obvious bleeding remission rapidly occurred in 11 patients. Thus, subsequent anticoagulation therapy was much safer than either anticoagulation therapy or hemostasis after bleeding remission, and LE-DVT was indeed alleviated or cured after colostomy and subsequent anticoagulation therapy in some patients according to our follow-up data. However, further studies must be conducted to investigate the effects of colostomy in hemorrhagic CRP patients with LE-DVT because of the small number of these patients until now.

However, our research has some weaknesses. One limitation is that only CRII patients with symptoms of DVT in the lower limbs were examined. Second, only hospitalized CRII patients were included, which inevitably involves patient selection bias. Third, this study is limited by the small number of CRII patients with LE-DVT and hemorrhagic CRII patients with LE-DVT treated by colostomy. Fourth, our study is limited by a lack of access to complete and long-term follow-up data on the treatment of CRII patients with LE-DVT. Further studies will be conducted to confirm our findings and further investigate the effect of colostomy in CRII patients with LE-DVT.

\section{Conclusion}

The incidence of LE-DVT was $5.3 \%$ in CRII patients after pelvic malignancy radiation, and approximately $15.5 \%$ of CRII patients had suspicious symptoms of LE-DVT in the lower limbs. A recent surgical history ( $\leq 6$ months), tumor recurrence or metastasis and a reduced $\mathrm{Hb}$ level were significantly associated with the development of LEDVT. These characteristics have potential as diagnostic markers to assist doctors in predicting the presence of LEDVT in CRII patients even if no suspicious symptoms of LE-DVT are observed. In addition, colostomy might be a good choice for treating intractable hemorrhagic CRII in patients with LE-DVT, in whom it was much safer to adopt anticoagulant therapy after colostomy, followed by obvious bleeding remission. This may assist the doctors in making clinical decisions when treating hemorrhagic CRII with LE-DVT.

\section{Abbreviations}

CRII, chronic radiation intestinal injury; LE-DVT, lower extremity deep venous thrombosis; CRP, chronic radiation proctitis; $\mathrm{BMI}$, body mass index; $\mathrm{Hb}$, hemoglobin, $\mathrm{BPC}$, platelet count; MPV, mean platelet volume; WBC, white blood cell; PT, prothrombin time; APTT, activated partial thromboplastin time; TT, thrombin time; FIB, fibrinogen; ALB, albumin; ROC, receiver operating characteristic; AUC, area under the receiver operating characteristic curve; OR, odds ratio; CI, confidence interval; IQR, interquartile range; $\mathrm{SD}$, standard deviation.

\section{Data Sharing Statement}

The data that support the findings of this study are available upon request from the corresponding author. The data are not publicly available due to privacy or ethical restrictions.

\section{Ethics Statement}

This study was approved by the Ethics Committee of the Sixth Affiliated Hospital of Sun Yat-sen University (No. 2018ZSLYEC-083) and was conducted in accordance with the provisions of the World Medical Association's Declaration of Helsinki of 1995 (revised in Tokyo, 2004). Because this was a retrospective study, the requirement for informed consent was waived. All authors declare that patient data are kept strictly confidential.

\section{Acknowledgments}

We would like to extend our sincere gratitude to every researcher and participant who contributed to this study. This study was supported by National Key Clinical Discipline, the Sun Yat-sen University Clinical Research 5010 Program (Grant Number: 2019021; Grant Number: 2017008), the National Natural Science Foundation of China (Grant Number: 81803163), the Natural Science Foundation of Guangdong Province (Grant Number: 2018A030310319) and Hospital Pharmacy Research Foundation of Guangdong Province (Grant Number: 2021A07). The funders played no role in the study design, data collection or analysis, decision to publish, or preparation of the manuscript. 


\section{Author Contributions}

All authors made significant contributions to the work reported, including the conception, study design and execution, data acquisition, analysis and interpretation, and drafting, revision or critically review of the article. All authors provided final approval of the version to be published, have agreed on the journal to which the article has been submitted; and agree to be accountable for all aspects of the work.

\section{Disclosure}

All authors declare that there are no conflicts of interest for this work.

\section{References}

1. Glover M, Smerdon GR, Andreyev HJ, et al. Hyperbaric oxygen for patients with chronic bowel dysfunction after pelvic radiotherapy (HOT2): a randomised, double-blind, sham-controlled phase 3 trial. Lancet Oncol. 2016;17:224-233. doi:10.1016/S1470-2045(15)00461-1

2. Sung H, Ferlay J, Siegel RL, et al. Global cancer statistics 2020: GLOBOCAN estimates of incidence and mortality worldwide for 36 cancers in 185 countries. CA Cancer J Clin. 2021;71:209-249. doi: $10.3322 /$ caac. 21660

3. Andreyev HJ, Vlavianos P, Blake P, et al. Gastrointestinal symptoms after pelvic radiotherapy: role for the gastroenterologist? Int J Radiat Oncol Biol Phys. 2005;62:1464-1471. doi:10.1016/j.ijrobp.2004. 12.087

4. Chen W, Zheng R, Baade PD, et al. Cancer statistics in China, 2015. CA Cancer J Clin. 2016;66:115-132. doi:10.3322/caac.21338

5. Hille A, Christiansen H, Pradier O, et al. Effect of pentoxifylline and tocopherol on radiation proctitis/enteritis. Strahlenther Onkol. 2005;181:606-614. doi:10.1007/s00066-005-1390-y

6. Sultania S, Sarkar R, Das K, Dhali GK. Argon plasma coagulation is an effective treatment for chronic radiation proctitis in gynaecological malignancy: an observational study. Colorectal Dis. 2019;21:465-471. doi:10.1111/codi.14541

7. Stacey R, Green JT. Radiation-induced small bowel disease: latest developments and clinical guidance. Ther Adv Chronic Dis. 2014;5:15-29. doi:10.1177/2040622313510730

8. Anwar M, Ahmad S, Akhtar R, et al. Antioxidant supplementation: a linchpin in radiation-induced enteritis. Technol Cancer Res Treat. 2017;16:676-691. doi:10.1177/1533034617707598

9. Kuku S, Fragkos C, McCormack M, Forbes A. Radiation-induced bowel injury: the impact of radiotherapy on survivorship after treatment for gynaecological cancers. Br J Cancer. 2013;109:1504-1512. doi:10.1038/bjc.2013.491

10. Krol R, Smeenk RJ, van Lin EN, et al. Systematic review: anal and rectal changes after radiotherapy for prostate cancer. Int $J$ Colorectal Dis. 2014;29:273-283. doi:10.1007/s00384-013-1784-8

11. Delaney G, Jacob S, Featherstone C, Barton M. The role of radiotherapy in cancer treatment: estimating optimal utilization from a review of evidence-based clinical guidelines. Cancer. 2005;104:1129-1137. doi:10.1002/cncr.21324

12. Andreyev J. Gastrointestinal symptoms after pelvic radiotherapy: a new understanding to improve management of symptomatic patients. Lancet Oncol. 2007;8:1007-1017. doi:10.1016/S14702045(07)70341-8

13. Bhutta BS, Fatima R, Aziz M. Radiation Enteritis. Treasure Island (FL): StatPearls; 2021.
14. Placer C, Lizarazu A, Borda N, et al. [Radiation proctitis and chronic and refractory bleeding. Experience with 4\% formaldehyde]. Cir Esp. 2013;91:111-114. Spanish. doi:10.1016/j.ciresp.2012.05.017

15. McDaid A, Logette E, Buchillier V, et al. Risk prediction of developing venous thrombosis in combined oral contraceptive users. PLoS One. 2017;12:e0182041. doi:10.1371/journal.pone.0182041

16. McLendon K, Attia M. Deep Venous Thrombosis (DVT), Risk Factors. Treasure Island (FL): StatPearls; 2018.

17. Zou PM, Li H, Cai JF, et al. A cohort study of incidences and risk factors for thromboembolic events in patients with idiopathic membranous nephropathy. Chin Med Sci J. 2018;33:91-99. doi:10.24920/11809

18. Guy JB, Bertoletti L, Magne N, et al. Venous thromboembolism in radiation therapy cancer patients: findings from the RIETE registry. Crit Rev Oncol Hematol. 2017;113:83-89. doi:10.1016/j. critrevonc.2017.03.006

19. Clarke-Pearson DL, Olt G. Thromboembolism in patients with Gyn tumors: risk factors, natural history, and prophylaxis. Oncology (Williston Park). 1989;3:39-45; discussion 45, 48.

20. Li Q, Xue Y, Peng Y, Li L. Analysis of risk factors for deep venous thrombosis in patients with gynecological malignant tumor: a clinical study. Pak J Med Sci. 2019;35:195-199. doi:10.12669/ pjms.35.1.365

21. Timp JF, Braekkan SK, Versteeg HH, Cannegieter SC. Epidemiology of cancer-associated venous thrombosis. Blood. 2013;122: 1712-1723. doi:10.1182/blood-2013-04-460121

22. Halle M, Ekstrom M, Farnebo F, Tornvall P. Endothelial activation with prothrombotic response in irradiated microvascular recipient veins. J Plast Reconstr Aesthet Surg. 2010;63:1910-1916. doi:10. 1016/j.bjps.2009.12.001

23. Kvolik S, Jukic M, Matijevic M, et al. An overview of coagulation disorders in cancer patients. Surg Oncol. 2010;19:e33-e46. doi:10.1016/j.suronc.2009.03.008

24. Roberge D, Skamene T, Nahal A, et al. Radiological and pathological response following pre-operative radiotherapy for soft-tissue sarcoma. Radiother Oncol. 2010;97:404-407. doi:10.1016/j.radonc. 2010.10.007

25. Wang PL, Cheng YB, Kuerban G. The clinical characteristic differences between thrombosis-related edema and lymphedema following radiotherapy or chemoradiotherapy for patients with cervical cancer. $J$ Radiat Res. 2012;53:125-129. doi:10.1269/jrr.11094

26. Olaf M, Cooney R. Deep venous thrombosis. Emerg Med Clin North Am. 2017;35:743-770. doi:10.1016/j.emc.2017.06.003

27. Coia LR, Myerson RJ, Tepper JE. Late effects of radiation therapy on the gastrointestinal tract. Int $J$ Radiat Oncol Biol Phys. 1995;31:1213-1236. doi:10.1016/0360-3016(94)00419-L

28. Yuan ZX, Ma TH, Wang HM, et al. Colostomy is a simple and effective procedure for severe chronic radiation proctitis. World J Gastroenterol. 2016;22:5598-5608. doi:10.3748/wjg.v22.i24.5598

29. Fowkes FJ, Price JF, Fowkes FG. Incidence of diagnosed deep vein thrombosis in the general population: systematic review. Eur J Vasc Endovasc Surg. 2003;25:1-5. doi:10.1053/ejvs.2002.1778

30. Zaghiyan KN, Sax HC, Miraflor E, et al. Timing of chemical thromboprophylaxis and deep vein thrombosis in major colorectal surgery: a randomized clinical trial. Ann Surg. 2016;264:632-639. doi:10. 1097/SLA.0000000000001856

31. Truong A, Hanna MH, Moghadamyeghaneh Z, Stamos MJ. Implications of preoperative hypoalbuminemia in colorectal surgery. World J Gastrointest Surg. 2016;8:353-362. doi:10.4240/wjgs.v8.i5.353

32. Saha P, Humphries J, Modarai B, et al. Leukocytes and the natural history of deep vein thrombosis: current concepts and future directions. Arterioscler Thromb Vasc Biol. 2011;31:506-512. doi:10.1161/ATVBAHA.110.213405

33. Zhang T, Li Q, Wang L, Li G. Expression variations and clinical significance of MMP-1, MMP-2 and inflammatory factors in serum of patients with deep venous thrombosis of lower extremity. Exp Ther Med. 2019;17:181-186. doi:10.3892/etm.2018.6922 
34. Singh G, Rathi AK, Singh K, Sharma D. Venous thromboembolism in cancer patients - magnitude of problem, approach, and management. Indian J Cancer. 2017;54:308-312. doi:10.4103/ijc.IJC_101_17

35. Mukai M, Oka T. Mechanism and management of cancer-associated thrombosis. J Cardiol. 2018;72:89-93. doi:10.1016/j.jjcc.2018.02.011

36. Donnellan E, Kevane B, Bird BR, Ainle FN. Cancer and venous thromboembolic disease: from molecular mechanisms to clinical management. Curr Oncol. 2014;21:134-143. doi:10.3747/co.21.1864

37. Kaiafa G, Savopoulos C, Kanellos I, et al. Anemia and stroke: where do we stand? Acta Neurol Scand. 2017;135:596-602. doi:10.1111/ ane. 12657
38. Hung SH, Lin HC, Chung SD. Association between venous thromboembolism and iron-deficiency anemia: a population-based study. Blood Coagul Fibrinolysis. 2015;26:368-372. doi:10.1097/ mbc. 0000000000000249

39. Evstatiev R. [Iron deficiency, thrombocytosis and thromboembolism]. Wien Med Wochenschr. 2016;166:437-446. German. doi:10.1007/ s10354-016-0514-6

\section{Publish your work in this journal}

Cancer Management and Research is an international, peer-reviewed open access journal focusing on cancer research and the optimal use of preventative and integrated treatment interventions to achieve improved outcomes, enhanced survival and quality of life for the cancer patient.
The manuscript management system is completely online and includes a very quick and fair peer-review system, which is all easy to use. Visit http://www.dovepress.com/testimonials.php to read real quotes from published authors. 